\title{
QCD predictions for polarised deep inelastic scattering accompanied by a forward jet in the low $x$ region of possible HERA measurements
}

\author{
J. KWIECIŃSKI,円 B. ZiAJA円 \\ Department of Theoretical Physics, \\ H. Niewodniczański Institute of Nuclear Physics, Cracow, Poland
}

\begin{abstract}
We estimate the cross-section and asymmetry relevant for the production of forward jets in polarised deep inelastic scattering in the region of small values of $x$ which can be probed at possible polarised HERA measurements. The kinematical cuts implemented in our analysis are the same as those which were used in the unpolarised deep inelastic scattering at HERA. The calculations are based on the double $\ln ^{2}(1 / x)$ resummation which controls the polarised deep inelastic scattering for small values of the Bjorken parameter $x$. We show that this resummation substantially enhances the corresponding cross-section and asymmetry. The predicted value of the asymmetry is found to vary between -0.01 and -0.04 within the small $x$ region which can possibly be probed at polarised HERA measurements.
\end{abstract}

The polarised deep inelastic scattering at small $x$ is dominated by the effects of the double $\ln ^{2}(1 / x)$ resummation [1, 2, 3]. Here, as usual, $x=Q^{2} /(2 p q)$, where $Q^{2}=$ $-q^{2}$ with $q$ and $p$ denoting the four momentum transfer between leptons and four momentum of the proton respectively. It has been pointed out in [4 that, apart from the structure function measurement, a process which probes the effects of double $\ln ^{2}(1 / x)$ resummation might be the deep inelastic scattering accompanied by a forward energetic jet. The idea of studying the deep inelastic ep scattering with an identified forward jet as a probe of the low $x$ behaviour of QCD was proposed by Mueller [5]. Since then it has been successfully applied to the case of unpolarised deep inelastic scattering (DIS) at HERA [6, 7, 8]. The Mueller's proposal was to study the unpolarised deep inelastic events $\left(x, Q^{2}\right)$ containing an identified forward jet $\left(x_{J}, k_{J}^{2}\right)$, where the longitudinal momentum fraction $x_{J}$ of the proton carried by a forward jet and jet

\footnotetext{
${ }^{1}$ e-mail: jkwiecin@solaris.ifj.edu.pl

2e-mail: beataz@solaris.ifj.edu.pl
} 
transverse momentum squared $k_{J}^{2}$ were assumed to fulfill the conditions :

$$
\begin{aligned}
& x_{J} \gg x, \\
& k_{J}^{2} \sim Q^{2} .
\end{aligned}
$$

The first condition $x_{J} \gg x$ guarantees that one probes the QCD dynamics in the low $x / x_{J}$ region (the quantities which are measured depend on the ratio $x / x_{J}$ ). The second condition $k_{J}^{2} \sim Q^{2}$ supresses the effects of the standard leading order (LO) DGLAP evolution (from $k_{J}^{2}$ to $Q^{2}$ ), and so one can probe the low $x$ QCD effects which go beyond this evolution. In the unpolarised DIS the forward jet measurement is therefore regarded as a useful tool for probing the BFKL pomeron [9]. Experimental results on forward jet production in deep inelastic scattering at HERA are presented in refs. [10, 11].

The BFKL pomeron decouples from polarised deep inelastic scattering. At low $x$ the latter is dominated by the novel effects of the double $\ln ^{2}(1 / x)$ resummation. The dominant contribution to this resummation is generated by ladder diagrams with quark (antiquark) and gluon exchange(s) along the ladder. The double logarithmic $\ln ^{2}(1 / x)$ terms come from the configuration of strongly ordered $x_{n}$ and $k_{n}^{2} / x_{n}$ [12], where $k_{n}^{2}$ and $x_{n}$ denote the transverse momenta squared and longitudinal momentum fractions of the proton exchanged along the parton ladder respectively. The effects of the double $\ln ^{2}(1 / x)$ resummation should therefore be still visible in the kinematical configuration $k_{J}^{2} \sim Q^{2}$. The effects of the conventional (LO) QCD evolution from the scale $k_{J}^{2}$ to $Q^{2}$ are on the other hand expected to be suppressed in this configuration.

In ref. [4] we have discussed in detail the differential structure function $x_{J} \frac{\partial^{2} g_{1}}{\partial x_{J} \partial k_{j}^{2}}$ describing (forward) jet production. It is linked to the corresponding differential crosssection in the standard way:

$$
\frac{\partial^{4} \sigma}{\partial x \partial Q^{2} \partial x_{J} \partial k_{J}^{2}}=\frac{8 \alpha_{e}^{2} \pi}{Q^{4}} \frac{\partial^{2} g_{1}}{\partial x_{J} \partial k_{J}^{2}} y(2-y),
$$

where $\alpha_{e}$ denotes the electromagnetic coupling constant, and $y$ describes the energy fraction of incoming electron carried by the interacting virtual photon, i.e. $y=q p /\left(p_{e} p\right)$ where $p_{e}$ denotes the for momentum of the incoming electron. In equation (3) we have omitted terms proportional to $\gamma^{2}=4 M^{2} x^{2} / Q^{2}$, where $M$ denotes nucleon mass, which are negligible at small $x$. The cross section in formula (3) corresponds, as usual, to the difference between the cross-sections for antiparallel and parallel spin orientations [13. Similarly as for the unpolarised case, restrictions (11), (2) applied to the polarised DIS forward jet events allow one to neglect the effects of DGLAP evolution and to concentrate on the low $x / x_{J}$ behaviour of $x_{J} \frac{\partial^{2} g_{1}}{\partial x_{J} \partial k_{J}^{2}}$. 
In this note we wish to study the cross-section itself, together with the asymmetry, taking into account the kinematical cuts which have recently been used in the forward jet measurements in unpolarised deep inelastic scattering at HERA [10, 11]. To be precise we shall give predictions for the cross-section $d \sigma / d x$ :

$$
\frac{d \sigma}{d x}=\int d k_{J}^{2} d x_{J} d Q^{2} \frac{\partial^{4} \sigma}{\partial x \partial Q^{2} \partial x_{J} \partial k_{J}^{2}}
$$

with the integration region restricted by the kinematical cuts which will be specified below. The asymmetry $A(x)$ is conventionally defined as:

$$
A(x)=\frac{d \sigma}{d x} /\left(2 \frac{d \sigma_{u}}{d x}\right),
$$

where $\frac{d \sigma_{u}}{d x}$ is the cross-section for forward jet production in unpolarised deep inelastic scattering.

The formula for the differential structure function can be written in the following form (see Fig. 1) :

$$
\begin{gathered}
x_{J} \frac{\partial^{2} g_{1}}{\partial x_{J} \partial k_{J}^{2}}= \\
\frac{<e^{2}>}{2} \bar{\alpha}_{s}\left(k_{J}^{2}\right) \sum_{i m} \Delta p_{i}\left(x_{J}, k_{J}^{2}\right) \Delta P_{m i}(0)\left[\Phi_{m}^{S}\left(\frac{x}{x_{J}}, k_{J}^{2}, Q^{2}\right)+\Phi_{m}^{N S}\left(\frac{x}{x_{J}}, k_{J}^{2}, Q^{2}\right)\right],
\end{gathered}
$$

where $\Delta p_{i}\left(x_{J}, k_{J}^{2}\right)$ are the (integrated) spin dependent parton distributions in the proton. The quantities $\Delta P_{i m}(0) \equiv \Delta P_{i m}(z=0)$, where $\Delta P_{i m}(z)$ (for quarks $\Delta P_{i m}(z)=$ $\left.\delta_{i m} \Delta P_{q q}\right)$ denote the LO splitting functions describing evolution of spin dependent parton densities. The indices $i$ and $m$ numerate quarks, antiquarks and gluons and the functions $\Phi_{m}^{S, N S}\left(\frac{x}{x_{J}}, k_{J}^{2}, Q^{2}\right)$ correspond to the singlet and non-singlet combinations of the spin dependent structure functions of the parton $m$. The average quark charge squared $<e^{2}>$ is defined as:

$$
<e^{2}>=\frac{1}{N_{f}} \sum_{i=1}^{N_{f}} e_{i}^{2},
$$

where $N_{f}$ denotes the number of quark flavours which we set equal to 3 . Finally, $\bar{\alpha}_{s}=$ $\alpha_{s} / 2 \pi$. Equation (6) was derived, assuming strong ordering of transverse momenta $\left(k_{i}^{2}<<k_{J}^{2} \sim k_{m}^{2}\right)$ and of the longitudinal momentum fractions $\left(x_{m}<<x_{i} \sim x_{J}\right)$ at the jet production vertex (see Fig. 1).

The functions $\Phi_{m}^{S, N S}\left(\frac{x}{x_{J}}, k_{J}^{2}, Q^{2}\right)$ are related to the singlet and the non-singlet combinations of unintegrated quark and antiquark distributions $f_{m}^{S, N S}\left(\frac{\bar{x}}{x^{\prime}}, k_{J}^{2}, k_{f}^{2}\right)$ in the parton 


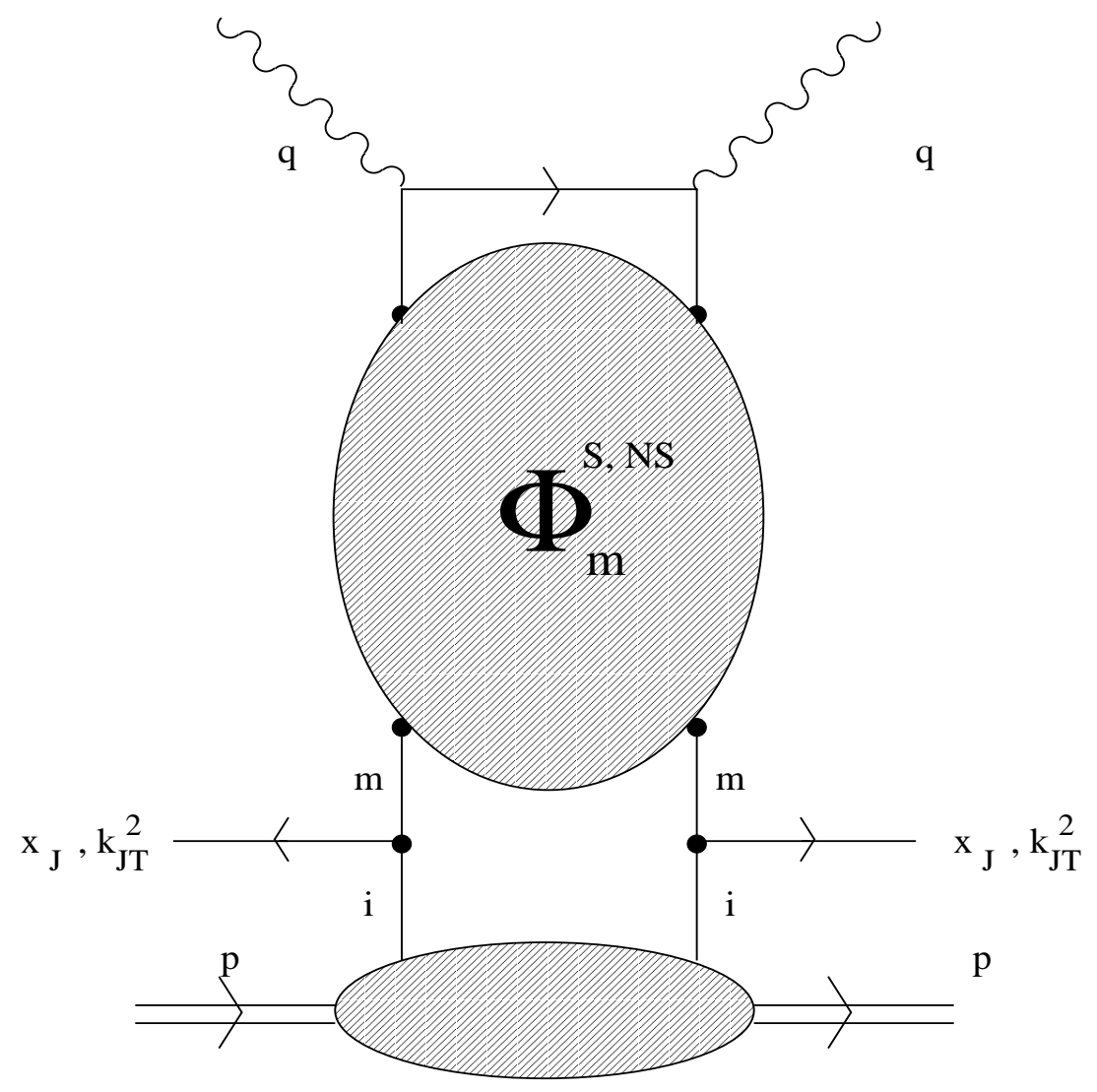

Figure 1: Diagrammatic representation of the formula (6) for the differential structure function $x_{J} \frac{\partial^{2} g_{1}}{\partial x_{J} \partial k_{J}^{2}}$ describing the forward jet production in polarised deep inelastic scattering.

$m$, where $k_{f}^{2}$ denotes the transverse momentum squared of the quark (antiquark) which is probed by the virtual photon:

$$
\Phi_{m}^{S, N S}\left(\frac{x}{x_{J}}, k_{J}^{2}, Q^{2}\right)=\int_{x_{\text {down }}}^{x_{u p}} \frac{d x^{\prime}}{x^{\prime}} \int_{k_{\text {down }}^{2}}^{k_{u p}^{2}} \frac{d k_{f}^{2}}{k_{f}^{2}} f_{m}^{S, N S}\left(\frac{\bar{x}}{x^{\prime}}, k_{J}^{2}, k_{f}^{2}\right),
$$

where $\bar{x}=x\left(1+\frac{k_{f}^{2}}{Q^{2}}\right)$. The integration limits $x_{u p}, x_{\text {down }}, k_{u p}^{2}$ and $k_{\text {down }}^{2}$ :

$$
\begin{aligned}
x_{\text {up }}=x_{J}, \quad x_{\text {down }} & =x\left(1+\frac{k_{J}^{2}}{Q^{2}}\right), \\
k_{\text {up }}^{2}=Q^{2}\left(\frac{x^{\prime}}{x}-1\right), & k_{\text {down }}^{2}=k_{J}^{2} /\left(\frac{x^{\prime}}{x}-\frac{k_{J}^{2}}{Q^{2}}\right)
\end{aligned}
$$

take into account restrictions implied by the phase-space limitation $k_{f}^{2}<Q^{2}\left(x_{J} / x-1\right)$ and by the ordering of longitudinal momentum fractions $x_{n}$ and of the ratios $k_{n}^{2} / x_{n}$ along the ladder diagrams defining the functions $f_{m}^{S, N S}\left(\xi, k_{J}^{2}, k_{f}^{2}\right)$ [4]. To be precise, the functions $f_{m}^{S, N S}\left(\xi, k_{J}^{2}, k_{f}^{2}\right)$ are the solutions of the integral equations which correspond to ladder diagrams in the double logarithmic $\ln ^{2}(1 / \xi)$ approximation. The functions $f_{m}^{N S}\left(\xi, k_{J}^{2}, k_{f}^{2}\right)$, 
with $m$ corresponding to $u, \bar{u}, d, \bar{d}$ are generated by ladder diagrams with quark (antiquark) exchange along the chain. The singlet combinations $f_{m}^{S}\left(\xi, k_{J}^{2}, k_{f}^{2}\right)(m=u, \bar{u}, d, \bar{d}, s, \bar{s}) \operatorname{mix}$ with the functions $f_{m}^{g}\left(\xi, k_{J}^{2}, k_{f}^{2}\right)$ describing the (spin dependent) gluon distribution in parton $m$. Both functions are generated by coupled integral equations corresponding to quark (antiquark) and gluon exchanges along the ladder. The integral equations for the functions $f_{m}^{S, N S, g}\left(\xi, k_{J}^{2}, k_{f}^{2}\right)$ resumming the double $\ln ^{2}(1 / \xi)$ terms are given in ref. [4]. The solutions of the integral equations for $f_{m}^{S, N S, g}\left(\xi, k_{J}^{2}, k_{f}^{2}\right)$ depend upon the scale $\mu^{2}$ which defines the coupling $\bar{\alpha}_{s}\left(\mu^{2}\right)$ controlling the elementary vertices along the partonic ladder. In ref. 化 we have considered two limiting cases which allow the analytic solution, i.e. $\mu^{2}=\left(k_{J}^{2}+Q^{2}\right) / 2$ and $\mu^{2}=k_{f}^{2} / \xi$. For the former choice of the scale the coupling does not change along the ladder while the latter case corresponds to the coupling which varies along the chain. The analytic expressions for the functions $f_{m}^{S, N S}\left(\xi, k_{J}^{2}, k_{f}^{2}\right)$ for both cases are given in ref. [⿴囗十 .

In order to calculate the cross-section $d \sigma / d x$ we have used the following kinematical cuts:

Tab. 1

\begin{tabular}{||c||}
\hline \hline$E_{e}^{\prime}>11 \mathrm{GeV}$ \\
\hline$y>0.1$ \\
\hline $160^{0}<\theta_{e}<173^{0}$ \\
\hline$x_{J}>0.035$ \\
\hline$k_{J}>3.5 \mathrm{GeV}$ \\
\hline $7^{0}<\theta_{J}<20^{0}$ \\
\hline$\frac{Q^{2}}{2}<k_{J}^{2}<2 Q^{2}$ \\
\hline \hline
\end{tabular}

We also set $E_{e}=30 \mathrm{GeV}$ and $E_{p}=820 \mathrm{GeV}$. The variables $E_{e}, E_{p}, E_{e}^{\prime}, \theta_{e}$ and $\theta_{J}$ denote the energy of the incoming electron, the energy of the incident proton, the energy of the scattered electron, the scattering angle of the electron and the angle of the measured jet respectively. The variables $x_{J}$ and $k_{J}^{2}$ denote as before the longitudinal momentum fraction $x_{J}$ of the proton carried by a forward jet and jet transverse momentum squared $k_{J}^{2}$. The angles $\theta_{e}$ and $\theta_{J}$ are measured with respect to the direction of the incident proton. The cuts summarised in Table 1 are the same as those which were used by the $\mathrm{H} 1$ collaboration in the analysis of forward jet production in unpolarised deep inelastic scattering [10] in the small $x$ region which can possibly also be probed at the polarised HERA measurements [14].

In Figures 2a - 2c we show our predictions for the spin dependent cross-section $d \sigma / d x$ plotted as the function of $x$. We present results corresponding to two choices of the scale $\mu^{2}$, i.e. $\mu^{2}=\left(k_{J}^{2}+Q^{2}\right) / 2$ (Fig. 2a) and $\mu^{2}=k_{J}^{2} / \xi$ (Fig. 2b) (the scale $\mu^{2}$ is the argument of the QCD coupling controlling the kernels of the integral equations for the functions $\left.f_{m}^{S, N S, g}\left(\xi, k_{J}^{2}, k_{f}^{2}\right)\right)$. In Fig. 2c we show our result which was obtained by neglecting the double $l n^{2}(1 / \xi)$ resummation effects. This approximation corresponds to neglecting the parton 
radiation between the forward jet and the virtual photon. It may be seen from the results shown in Figures 2a-2c that the impact of the double $\ln ^{2}(1 / \xi)$ resummation effects on the cross-section is very significant. These effects give the cross-section which rises much more steeply with decreasing $x$, down to $x \sim 5 \cdot 10^{-4}$, than the cross-section which corresponds to the case where the double $\ln ^{2}(1 / \xi)$ effects were neglected. At their maximum the two cross-sections differ by a factor equal to about 20 or 40 , depending upon the choice of the scale $\mu^{2}$. The decrease of the cross-section with decreasing $x$ in the region $x<5 \cdot 10^{-4}$ is a kinematical effect which follows from the cuts (see Tab. 1).

In Fig. 3 we show predictions for the asymmetry $A(x)$ defined by equation (5). The asymmetry $A(x)$ was obtained by combining our results for $d \sigma / d x$ with the results of the calculation of the cross-section $d \sigma_{u} / d x$ describing the jet production in unpolarised deep inelastic scattering with the same kinematical cuts as those summarised in Tab. 1 [8]. The presented asymmetry $A(x)$ weakly decreases with decreasing $x$. It is found to be approximately equal to -0.01 for $x=0.004$ and to decrease down to -0.038 for $\mu^{2}=\left(k_{J}^{2}+Q^{2}\right) / 2$ or to -0.022 for $\mu^{2}=k_{f}^{2} / \xi$ at $x=0.0005$.

To sum up, we have estimated in this note the impact of the double $\ln ^{2}(1 / x)$ effects on the corresponding cross-sections and asymmetries describing the forward jet production in polarised deep inelastic lepton scattering in the small $x$ regime which can be probed at the possible polarised HERA measurements. We have found that double logarithmic effects substantially enhance the absolute magnitude of the corresponding cross-section and of the asymmetry. These quantities would be negligibly small if the double logarithmic effects were neglected. The asymmetry parameter $A(x)$ was found to vary between -0.01 and -0.04 at the small $x$ range relevant for the possible polarised HERA measurements.

\section{Acknowledgments}

We thank John Outhwaite for providing us with the results of the calculation of $d \sigma_{u} / d x$. This research has been supported in part by the Polish Committee for Scientific Research grant 2 P03B 8913 and by the EU Fourth Framework Programme 'Training and Mobility

of Researchers', Network 'Quantum Chromodynamics and the Deep Structure of Elementary Particles', contract FMRX-CT98-0194.

\section{References}

[1] J. Bartels, B.I. Ermolaev and M.G. Ryskin, Z. Phys. C70 (1996) 273.

[2] J. Bartels, B.I. Ermolaev and M.G. Ryskin, Z. Phys. C72 (1996) 627. 
[3] J. Kwieciński, B. Ziaja, Phys. Rev. D 60 (1999) 054004.

[4] J. Kwieciński, B. Ziaja, hep-ph/9906499, to appear in Phys. Lett. B.

[5] A. Mueller, Nucl. Phys. B (Proc. Suppl.) 18 C (1990) 125; J. Phys. G 17 (1991) 1443.

[6] J. Bartels, A. DeRoeck, M. Loewe, Z. Phys. C 54 (1992) 635; W.K. Tang, Phys. Lett. B 278 (1992) 363; J. Bartels et al., Phys. Lett. B384 (1996) 300.

[7] J. Kwieciński, A.D. Martin, P.J. Sutton, Phys. Rev. D46 (1992) 921; Phys. Lett. B287 (1992) 254.

[8] J. Kwieciński, A.D. Martin, J. Outhwaite, Eur. Phys. J. C9 (1999) 611.

[9] E.A. Kuraev, L.N.Lipatov and V.S. Fadin, Zh. Eksp. Teor. Fiz. 72 (1977) 373 (Sov. Phys. JETP 45 (1977) 199); Ya. Ya. Balitzkij and L.N. Lipatov, Yad. Fiz. 28 (1978) 1597 (Sov. J. Nucl. Phys. 28 (1978) 822); J.B. Bronzan and R.L. Sugar, Phys. Rev. D17 (1978) 585; T. Jaroszewicz, Acta. Phys. Polon. B11 (1980) 965; L.N. Lipatov, in "Perturbative QCD", edited by A.H. Mueller, (World Scientific, Singapore, 1989), p. 441; L.N. Gribov, E.M. Levin and M.G. Ryskin, Phys. Rep. 100 (1983) 1.

[10] H1 Collaboration, C. Adloff et al., Nucl. Phys. B538 (1999) 3.

[11] ZEUS Collaboration, Eur. Phys. J. C 6 (1999) 239.

[12] V.G. Gorshkov et al., Yad. Fiz. 6 (1967) 129 (Sov. J. Nucl. Phys. 6 (1968) 95); L.N. Lipatov, Zh. Eksp. Teor. Fiz. 54 (1968) 1520 (Sov. Phys. JETP 27 (1968) 814.

[13] Spin Muon Collaboration (SMC), D. Adams et al., Phys. Rev. D56 (1997) 5330; B. Adeva et al. (SMC), CERN-PPE/97-118; SMC, D. Adams et al., Phys. Lett. B396, 338 (1997).

[14] A. De Roeck, Proceedings of the Cracow Epiphany Conference on Spin Effects in Particle Physics, Kraków, Poland, January 9-11, 1998, Acta Phys. Polon. B29 (1998) 1343. 

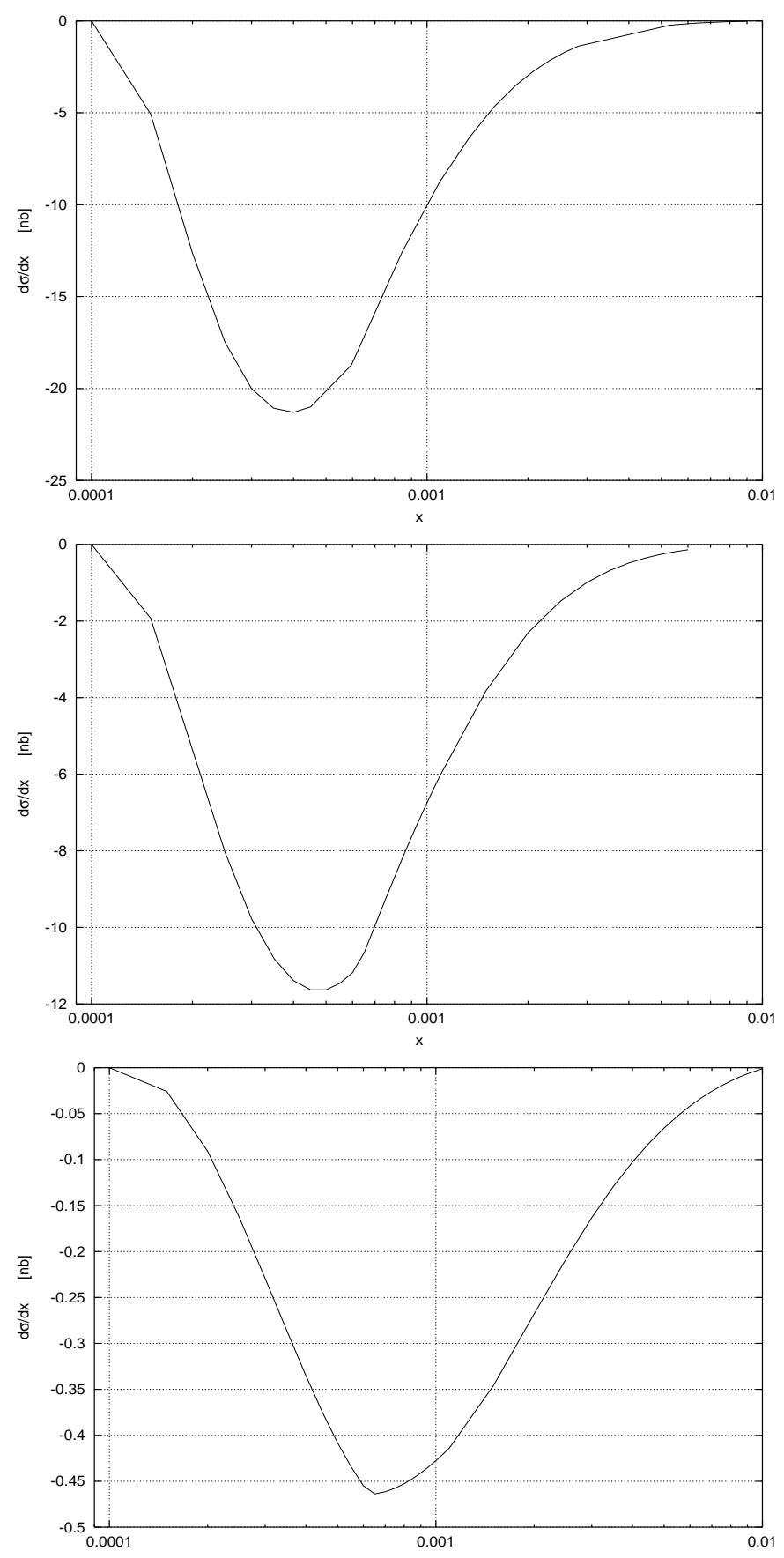

Figure 2: The cross-section $\frac{d \sigma}{d x}$ for forward jet production in polarised deep inelastic scattering plotted as the function of $x$. Figures $2 \mathrm{a}$ and $2 \mathrm{~b}$ show results which include the double logarithmic $\ln ^{2}(1 / \xi)$ effects and correspond to two choices of the scale $\mu^{2}$ which appears as the argument of the QCD coupling controlling the kernels of the corresponding integral equations for the functions $f_{m}^{S, N S, g}\left(\xi, k_{J}^{2}, k_{f}^{2}\right)$. Fig. 2a corresponds to $\mu^{2}=\left(k_{J}^{2}+Q^{2}\right) / 2$ and Fig. $2 \mathrm{~b}$ to $\mu^{2}=k_{f}^{2} / \xi$ respectively. Fig. 2c shows the crosssection $\frac{d \sigma}{d x}$ in the approximation when the double logarithmic resummation is neglected. 


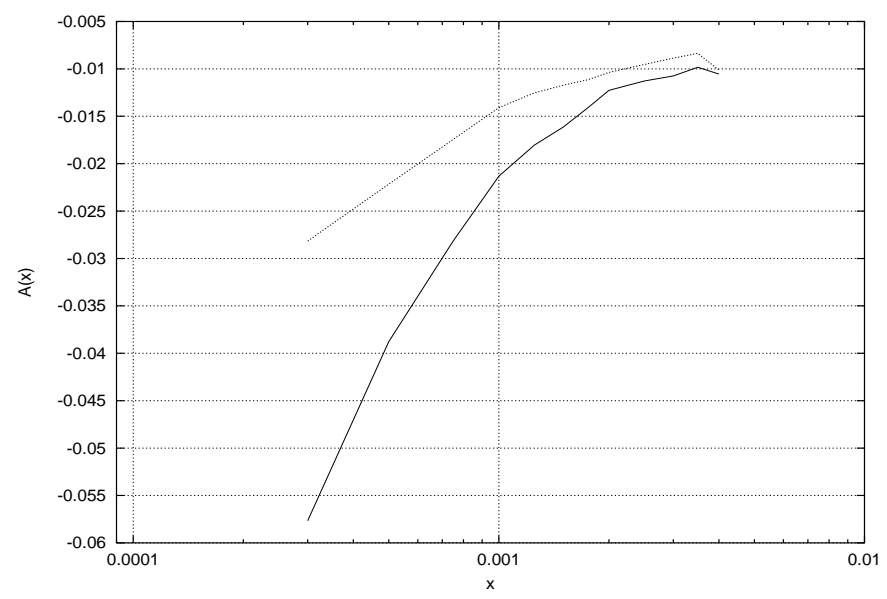

Figure 3: The asymmetry parameter $A(x)$ defined by eq. (5). The two curves correspond to two choices of the scale $\mu^{2}$ which appears as the argument of the QCD coupling controlling the kernels of the corresponding integral equations for the functions $f_{m}^{S, N S, g}\left(\xi, k_{J}^{2}, k_{f}^{2}\right)$. The lower and upper curves correspond to $\mu^{2}=\left(k_{J}^{2}+Q^{2}\right) / 2$ and to $\mu^{2}=k_{f}^{2} / \xi$ respectively. 